\title{
Experimental Investigation of Three-Dimensional Flow Development by Pitching Low-Aspect-Ratio Wing
}

\author{
Huai-Te $\mathrm{Yu}^{1}$ \\ Chung Cheng Institute of Technology, National Defense University, Taoyuan, Taiwan (R.O.C.) \\ and \\ Luis P. Bernal ${ }^{2}$ \\ University of Michigan, Ann Arbor, MI, 48109, U.S.A.
}

\begin{abstract}
This paper reports stereo flow evolution and aerodynamics of a pitching wing in water channel from zero to 45 -degree pitch angle in still water and reduced pitch rate of 0.39 , and earlier stage of transition flow at maximum angle of attack. The wing is a rectangular flat plate with aspect ratio of four at leading-edge pivot. Wall effect on the pitching wing and stationary wing in test environment is examined by means of direct force measurement. The stereo image is captured through two cameras together with PIV system; comparisons among two camera images and stereo images are provided. We ascertain that twodimensional flow features significant aerodynamic forces during the pitching maneuver, and therefore encourages lift generation, which mechanism includes the formation of Starting vortex and Leading-edge vortex together with Pivot vortex. However, the formations of Secondary vortex and Stopping vortex apparently are malignant to the lift generation at high angle of attack. Also revealed in the paper is that the formation of Tip vortex causes a promising drag, and induces detrimental spanwise flow to Leading-edge vortex on span locations other than wingtip, transiting aerodynamic forces decreasingly to steady state. Moreover, the trajectory of vortexes is documented.
\end{abstract}

\section{Nomenclature}

$\begin{array}{ll}A R & =\text { geometric aspect ratio }(=2), b^{2} / S, \mathrm{~m} \\ A R_{\text {eff }} & =\text { effective aspect ratio, } 2^{*} A R, \mathrm{~m} \\ b & =\text { wing wetted span, } 2^{*} c, \mathrm{~m} \\ C_{D} & =\text { drag coefficient, } 2 * D / \rho U_{\infty}^{2} S, 1 \\ C_{L} & =\text { lift coefficient, } 2^{2} L / \rho U_{\infty}{ }^{2} S, 1 \\ c & =\text { wing mean chord }(=5.08 \mathrm{~cm}), \mathrm{m} \\ c_{t} & =\text { wing tip chord, } \mathrm{m} \\ c_{r} & =\text { wing root chord, } \mathrm{m} \\ D & =\text { drag force, } \mathrm{N} \\ e & =\text { relaxation coefficient, } 1 \\ F_{A} & =\text { axial force, } \mathrm{N} \\ F_{N} & =\text { normal force, } \mathrm{N} \\ F x & =X \text { component of force in sensor frame of reference, } \mathrm{N} \\ F y & =Y \text { component of force in sensor frame of reference, } \mathrm{N} \\ h & =\text { hold parameter, } 1 \\ K & =\text { reduced pitch rate, } c \alpha_{m}^{\prime} / 2 U_{\infty}, 1 \\ L & =\text { lift force, } \mathrm{N} \\ v & =\text { kinematic viscosity, } \mathrm{m}^{2} / \mathrm{s}\end{array}$

\footnotetext{
${ }^{1}$ Assistant Professor, Department of Mechanical and Aerospace Engineering, Chung Cheng Institute of Technology, National Defense University, htyu0504@ndu.edu.tw, and AIAA Member.

${ }^{2}$ Professor, Department of Aerospace Engineering, University of Michigan, 1pb@umich.edu, and AIAA Member.
} 


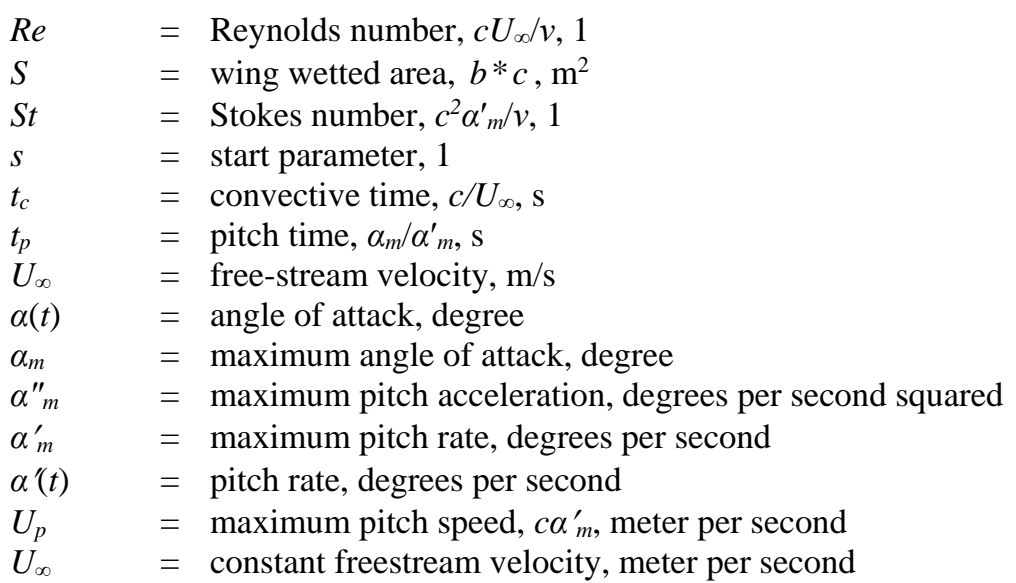

\section{Introduction}

$\mathrm{P}$ ching airfoils and wings are a canonical case in studying unsteady aerodynamics with relevant application to post-stall maneuvers of manned aircraft and other maneuvers of manned, unmanned and bio-inspired flight that has been the subject of research since the 1900s until today ${ }^{1-17}$. Most of experimental work in the past considered two-dimensional airfoils, such as NACA 0015 and NACA0012; very few work employed finite-aspectratio wings. For a pitching airfoil, the low pressure on wing leeward surface increases the lift during the motion, which is considerably associated with the formation of a Leading-edge vortex. As a finite-aspect-ratio wing is considered, the Tip vortex would typically induce drag force on the wing and causes less lift force at given angle of attack.

Recently, the development of micro aerial vehicles (MAVs) promotes the degree of rapid pitching wings for dust response and superior maneuver, which is also applicable for the perching of aerial vehicles. Using rectangular flat plates, Yilmaz et al. (2010) show scaling behaviors at phases higher than 20 degrees by means of dye injection, shifted with respect to the convective time based on reduced pitch rate $K$ for the wing at quarter-chord pivot axis. Yu and Bernal (2013) reported systematic force data for a range of $K\left(=c \alpha^{\prime}{ }_{m} / 2 U_{\infty}\right)$ from 0.022 to $\infty$ (Mathematically speaking, the $K$ equal to infinity is incorrectly representative of pitching wings in still water, which is a condition similar to flyers in hovering mode), and employ two-dimensional PIV technology to capture dynamic flow at geometrical mid-span and three-quarter span. They reported the flow is favorably two-dimensional for reduced pitch rate higher than 0.39 and lower pitch angle. They also find that the pivot-axis location determines the origination of Starting vortex, which changes the pressure distribution on the wing, but does not introduce three-dimensional flow. Yu et al. (2013) also report force data for several flat-plate wing geometries at various pivot axes for $K=0.39$; they find wing planform geometry has less influence on aerodynamic forces than reduced pitch rate even though the spanwise flow is more significant. Recent preliminary experimental work for two-dimensional airfoils are reported by Ol (2009), Ol et al. (2010), Grandlund et al. (2010, 2011a-b, 2013), and Baik et al. (2010).

In this study, firstly, we investigate the influence of wall effect on a pitching rigid wing using direct force detection technique for the facility in use. Secondly, we use lens-shift Stereoscopic Particle Image Velocimetry (SPIV) to measure the vortical structures and out-of-plane velocity field on planes at three spanwise locations, which are mid-span, three-quarter span, and wingtip. The purpose is to understand the development of Tip vortex for constant pitch-rate wing and its interaction with the Leading-edge vortex, and to determine the relation between vortical structure, spanwise flow and aerodynamic forces. The use of a rigid rectangular flat plate with effective aspect ratio of four is considered.

\section{Experimental Setup}

\section{A. Wing Configuration and Water Channel}

The wing is a rectangular flat plate with effective aspect ratio of four, and was tested in the low-turbulence freesurface water channel at University of Michigan, which has $61 \mathrm{~cm}$ width and nominal depth of $57 \mathrm{~cm}$. The constant freestream velocity is controlled through an AC induction motor and water depth within a range from $5 \mathrm{~cm} / \mathrm{s}$ to 40 $\mathrm{cm} / \mathrm{s}$. The wing is made of clear acrylic sheet, and has two-inch chord and two-chord span immersed in the water. 
The wing thickness is 0.125 ", equivalent to $6.25 \%$ of wing chord. All edges in the water are all rounded and only leading-edge pivot configuration is considered.

\section{B. Wing Kinematics}

The wing motion is a linear pitch ramp from $0^{\circ}$ to $45^{\circ}$. To minimize model vibration at the start and stop of the pitching, smoothing maneuver was operated using a smoothing function defined by Eq. (1), describing regions of constant pitch rate and pitch acceleration. As a result, fascinating regions of maximum pitch angle $\left(\alpha_{m}\right)$, constant pitch rate $\left(\alpha_{m}^{\prime}\right)$, and pitch acceleration $\left(\alpha^{\prime \prime}\right)$ are sufficed.

$$
\alpha(t)=\frac{\alpha_{m}}{2 B} \sum_{i=1}^{4}(-1)^{i+1} \ln \left\{\cosh \left[B\left(t-t_{i}\right) / t_{p}\right]\right\}, i=1,2,3,4
$$

where

$$
B=2 t_{p} / t_{a} .
$$

The parameter $B$ is a ratio of pitch time $\left(t_{p}=\alpha_{m} / \alpha_{m}^{\prime}\right)$ to pitch acceleration time $\left(t_{a}=\alpha_{m}^{\prime} / \alpha_{m}^{\prime \prime}\right)$. The parameter $B$ can be obtained by specifying parameters $\beta$ and $\gamma$. The $\beta$ is a ratio of smoothing angle to the maximum angle of attack; the $\gamma$ is a ratio of margin acceleration to the maximum acceleration during the smoothing region.

There are five phases to implement the wing motion, which includes start phase, pitch-up phase, hold phase, pitch-return phase, and relaxation phase. The duration for either pitch-up phase or pitch-return phase is the same. For force measurement, the duration of the start phase is one convective time; the hold duration is at least 130 convective times $(h>130)$ to ensure the flow reaching steady conditions at maximum pitch angle of $45^{\circ}$; the relaxation duration is about 30 convective times $(e>30)$ to return the flow to the undisturbed initial condition. For SPIV measurements, the time for relaxation is at least 30 convective time or pitch time.

In order to achieve maximum pitch rate, two rotary tables by Velmex were used to pitch the wing, which are B4818TS (for Kinematics I) and B4836TS (for Kinematics II), as shown in Table 1. Each rotary table is driven by the same model of stepper motor RK266-03. There are 13 points within every smoothing angle. For all measurements the motion is repeated 60 times and then phase-averaged approach is employed to obtain mean values with relatively small uncertainty. For detail implementation of wing kinematics, please see Yu et al. (2013), and Yu and Bernal (2013).

\begin{tabular}{|c|c|c|c|c|c|c|c|c|c|c|c|c|c|c|}
\hline $\begin{array}{l}\text { Kine- } \\
\text { matics }\end{array}$ & $\alpha_{m}^{\prime}$ & $B$ & $t_{p}$ & $t_{a}$ & $\alpha^{\prime \prime}$ & $(\Delta \alpha)$ & $\gamma$ & & $\alpha_{m}$ & $U_{\infty}$ & $\tau=t_{p} / t_{c}$ & $2 t_{d} / t_{c}$ & $\alpha_{m}^{\prime \prime} c^{2} / U_{\infty}^{2}$ & $K$ \\
\hline I & 15 & 1 & 0 & 0 & 7 & 0 & & & & $\begin{array}{c}0 \\
17.5\end{array}$ & $\begin{array}{l}- \\
1\end{array}$ & & & \\
\hline II & 76.4 & 21.60 & 0. & 0.109 & 1402 & 0.13 & & & & $\begin{array}{c}0 \\
8.6\end{array}$ & - & $\begin{array}{c}- \\
0.184\end{array}$ & 8.54 & $\begin{array}{c}- \\
0.39\end{array}$ \\
\hline
\end{tabular}

Table 1 Parameters for wing kinematic generation and freestream condition.

Unit of $\alpha_{m}^{\prime}$ is degree per second, $t_{p}$ is second, $t_{a}$ is second, $\alpha_{m}^{\prime \prime}$ is degree per second squared, $\Delta \alpha$ is degree, $c$ is inch, $\alpha_{m}$ is degree, $U_{\infty}$ is centimeter per second.

\section{Direct Force Measurement and Data Processing}

The wing was attached to the tool side of force sensor through a sensor adapter by screwing into a position where three-hole pattern on the wing being designed to align with the pitch axis and coincident with axes of the force sensor. And then fasten to a rotary table. The force sensor is a Nano 43 Force/Torque sensor manufactured by ATI Industrial Automation, which measures forces in three directions, including the chord-wise direction (x-axis), normal to the wing surface (y-axis) and in the span-wise direction (z-axis), and also corresponding torques. The sensor's maximum calibrated load is $18 \mathrm{~N}$ and the resolution is $1 / 256 \mathrm{~N}$, in all three axes. Both the force sensor and the rotary table are positioned above the water surface and in the air. The sensor adapter is made of aluminum and designed to minimize the mass of the system. The total mass attached to the sensor is no more than 46.2 grams, which includes the wing itself, a sensor adapter and screws. Because of the small mass, inertia forces and static weight of the model are very small, which makes static calibration unnecessary. The forces measured by the sensor 
( $F x$ and $F y$ ) are first converted to axial and normal forces, $F_{A}$ and $F_{N}$, and then to components in the laboratory frame of reference for lift force $(L)$ and drag force $(D)$ and their corresponding force coefficients.

The processing of force data includes an application of low-pass filter and a tare procedure. In order to avoid phase distortion, to reduce spurious oscillations introduced by the filter, and to provide sufficient noise attenuation, the low-pass filter is determined to be a zero-phase first-order two-path Butterworth filter. The cutoff frequency of the filter was chosen according to Fourier transform analysis of the motion acceleration to retain $90 \%$ of spectrum content. Therefore, the cutoff frequency for Kinematics I and II is 8.76 and $8.71 \mathrm{~Hz}$, respectively. The noise introduced by the rotary table and stepper motor is in a range of $100 \mathrm{~Hz}$ to $600 \mathrm{~Hz}$, and the wing vibration fundamental frequency in air and in still water is $50 \mathrm{~Hz}$ and $15 \mathrm{~Hz}$, respectively. These noises are significantly higher than the filter cutoff frequency.

The tare procedure is used to isolate the hydrodynamic force from model inertial and model weight contributions to the measured force. This includes static tares and dynamic tares. The static tares are measurements in air and still water at fixed angle from $0^{\circ}$ to maximum angle of attack 45 degrees with an increment of 3 degrees. The dynamic tares are measurements in air with the same kinematics as in the flow experiments. In addition, the force measurements at fixed angle of attack in steady flow are also considered. The kinematics is similar to static tare measurements but with a longer hold duration at the free-stream velocity of interest. In all force measurements, the same filter cutoff frequency was used.

All force measurements are repeated 60 times from $0^{\circ}$ to fixed angle of attack with sampling rate of $5,000 \mathrm{~Hz}$, and ensemble averages are reported. It is found that the present wing configuration yields negligible static tares because of the very small mass of the wing and mounting hardware.

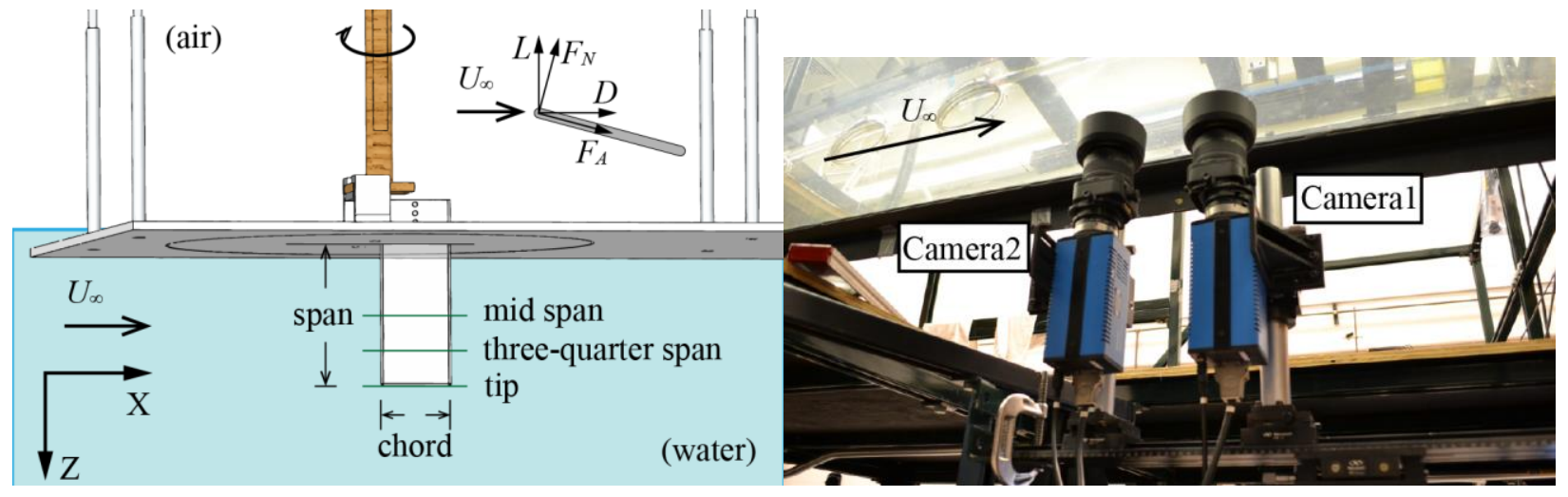

Fig. 1 Wing configurations for PIV system.

\section{Lens-Shift Stereoscopic PIV}

The configuration of lens-shift stereo particle image velocimetry (SPIV) is shown in Figs. 2-3, for coordinates on $\mathrm{X}-\mathrm{Z}$ plane and $\mathrm{Y}-\mathrm{Z}$ plane. The distance from object plane to lens plane is denoted by $p$, and the distance from image plane to lens plane is denoted by $q$. Two cameras were used to capture particle displacements in object plane where laser sheet is placed, which coordinates are denoted by X, Y, and Z. The subscripts, such as 1 and 2, represent data from Camera 1 and Camera 2, respectively. According to Figs. 2-3, Camera 1 is placed practically at downstream location whereas Camera 2 is placed at an upstream location. Both cameras were mounted with perspective control lens, PC-E Micro NIKKOR, to accomplish the offset of lens axis from image axis. The supplemental description of present SPIV system can be found in [29], the highlight of the system is given below.

Assume two cameras behave in a similar manner and $p \gg Z$, three components of particle displacement on object plane could be found using Eqs. (2)- (4).

$$
\begin{gathered}
d X=d x_{1} \frac{p_{1}-d Z}{p_{1} M_{1}}-d Z \frac{X-\Delta X_{1}}{p_{1}} \\
d Y=d y_{1} \frac{p_{1}-d Z}{p_{1} M_{1}}-d Z \frac{Y}{p_{1}}
\end{gathered}
$$




$$
d Z=\left(\frac{d x_{2}}{M_{2}}-\frac{d x_{1}}{M_{1}}\right) /\left[\frac{X-\Delta X_{2}}{p_{2}}-\frac{X-\Delta X_{1}}{p_{1}}+\frac{d x_{2}}{M_{2} p_{2}}-\frac{d x_{1}}{M_{1} p_{1}}\right]
$$

where the parameters of $M_{1}, M_{2}, p_{1}, p_{2}, \Delta X_{1}$, and $\Delta X_{2}$ are determined during calibration process; the values used in present study are given in Table 2, as well as the corresponding uncertainties. As shown in Table 2, both cameras give very similar magnification factors.

After calibration, there are three steps to evaluate particle velocity. The first step is to obtain particle displacements on the film of individual camera (such as $d x_{1}$ and $d y_{1}$ for Camera 1, and $d x_{2}$ and $d y_{2}$ for Camera 2) using cross-correlation technology, which is a general approach used for conventional two-dimensional PIV system. The second step is to evaluate particle displacements in Cartesian coordinates in illuminated plane (or object plane) based on the results from the first step. Finally, particle velocity $V$ in three components is determined using Eq. (5) by introducing $d t$, a time difference between interrogation windows. One of the windows has low resolution and the other has high resolution with a displacement by $d t$. In this study, three-pixel particle displacement based on constant freestream velocity $\left(U_{\infty}\right)$ or maximum pitching speed $\left(U_{p}=c \alpha_{m}^{\prime}\right)$ was used to estimate $d t$. The size of interrogation window of low resolution and high resolution is $64 \times 64$ and $32 \times 32$ pixels, respectively. The spatial resolution is about $1 \mathrm{~mm}$.

$$
\vec{V}=(d \vec{X}+d \vec{Y}+d \vec{Z}) / d t
$$

The thickness of laser sheet serves as a maximum limit of particle velocity in Z-component and was employed to verify the quality of PIV data. Presented in this paper are data evaluated according to laser thickness of $1.8 \mathrm{~mm}$; all data were affirmed that values of out-of-plane velocity are within laser thickness. Moreover, PIV images were taken at horizontal planes perpendicularly spreading through three locations on geometric wing span, including mid-span, three-quarter span, and wingtip, shown as red lines in Fig. 1. Since the laser sheet was kept at the same horizontal plane during the experiment, the wing model was adjusted vertically to desired span locations. Hence, the water level of the channel has to be filled or drained to response to the amount of vertical change of wing model.

Table 2 Parameters for lens-shift stereo PIV system.

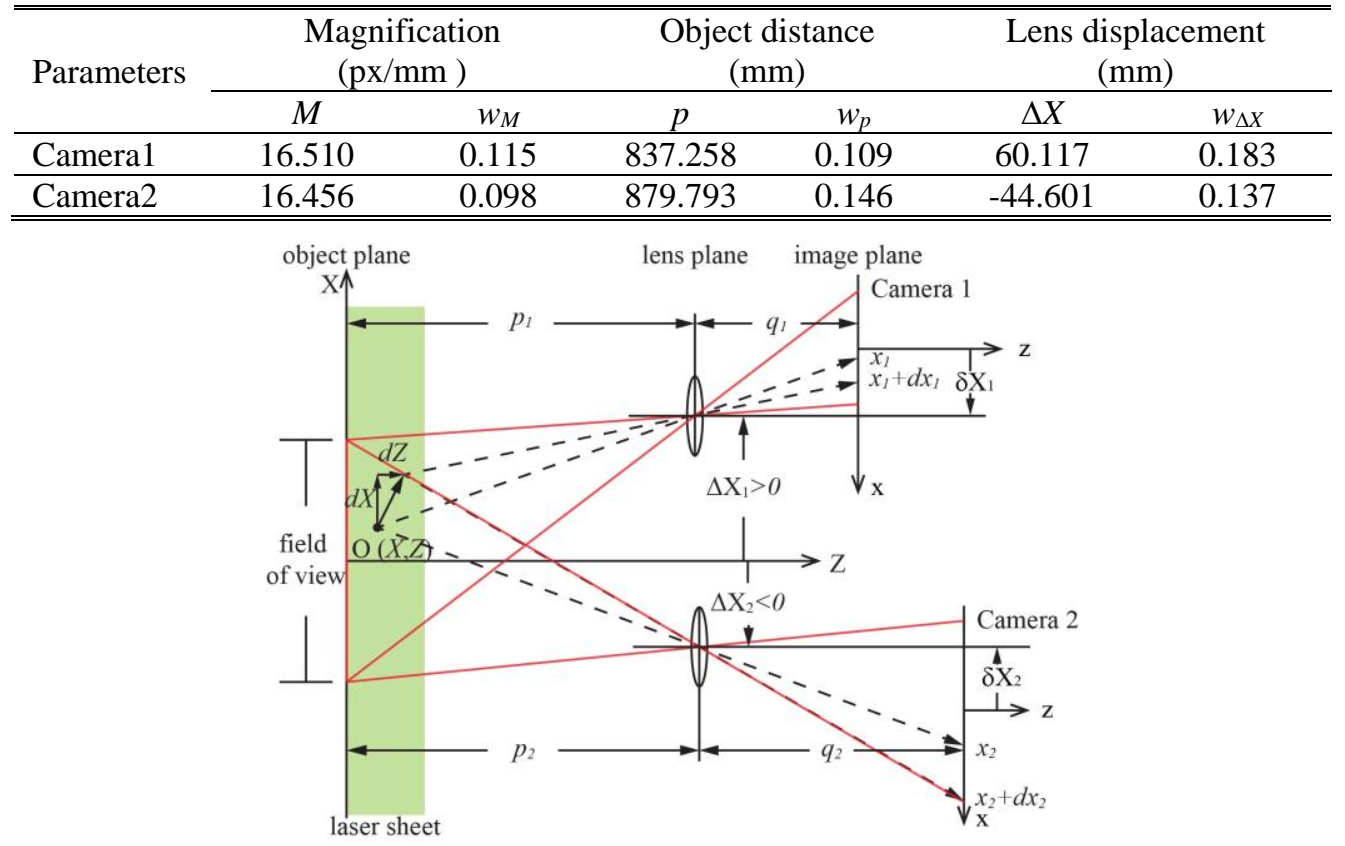

Fig. 2 Schematics of a lens-shift Stereo PIV geometry in the x-z plane.

As a result, stereo PIV images of vorticity and out-of-plane velocity are documented. The vorticity is dimensionless with pitching time whereas out-of-plane velocity is dimensionless with maximum pitching speed or freestream velocity. Both are contoured with streamlines at phases of interest, whose values are assigned to a colormap in a scale of uncertainty of $8 \mathrm{~s}^{-1}$ for vorticity and $4.5 \mathrm{~cm} / \mathrm{s}$ for out-of-plane velocity. The maximum and 
minimum values of the quantities of interest, within the field of view of display but outside wing shadow, are labeled to the colorbar shown in the figures. The shadow of the wing was identified from outlines of overlay regions of wing images on both Camera1 and Camera 2 using Adobe Illustrator, and presented in transparent gray area in PIV data. Since the edges of the wing were colored by black marker, the shadow due to marked leading edge and trailing edge under the illuminated light from the upper edge of the display are also identified and shown. Additionally, results from Camera1 and Camera2 were processed in a manner similar to that by combining results from two cameras, i.e., the median filter was applied to the data averaged over nominal 60 image-samples, taking no account of failure data points determined using cross-correlation technology.
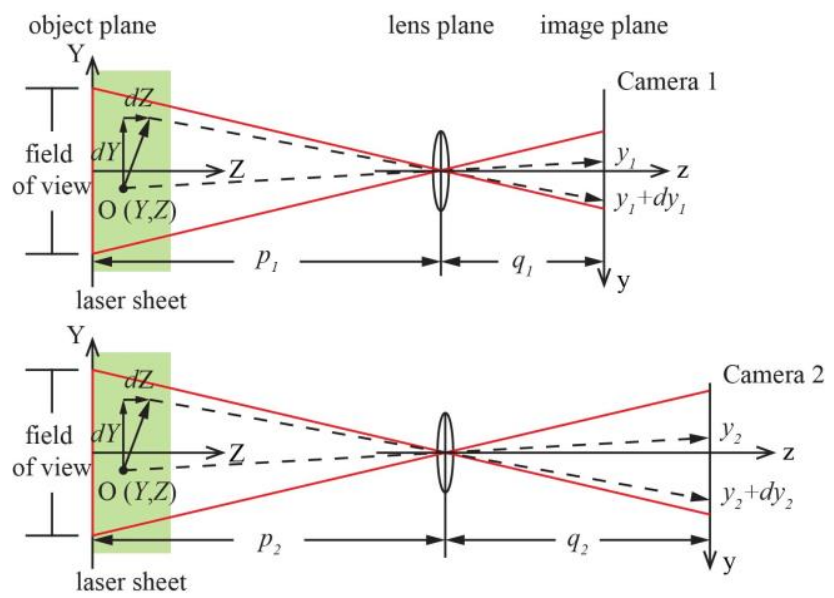

Fig. 3 Schematics of a lens-shift Stereo PIV geometry in the y-z plane.

\section{Results and Discussions}

\section{A. Investigation on Wall Effect}

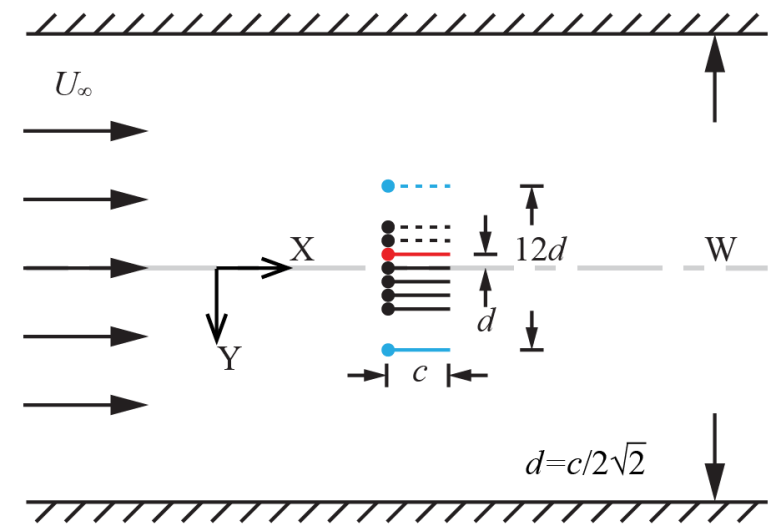

Fig. 4 Schematic drawing of a pitching wing at various initial positions to study side wall effect. The wing position is drawn to scale with respect to water channel width, but wing chord is not drawn to scale. The solid circle represents pivot axis location.

To investigate effects from the sidewall the force measurements were conducted at several wing initial positions across the channel, as shown in Fig. 4, which may interfere with force evolutions dynamically or statically [17]. There are nine positions considered, four positions are located upward from the center of the channel and four positions are translated downward from the center of the channel. The smallest distance between these positions is the projected length of wing chord by half and denoted by $d$. The farthest distance is $6 d$ away from the center, which gives about 3.8 chord-lengths close to the wall. The red solid curve represents the wing position where our most experiments were conducted as the configuration of leading-edge pivot was concerned, such that the wing centerchord is positioned at the center of the channel at $\alpha_{m}=45^{\circ}$. The upper positions are presented in dotted curves, and lower positions are presented in solid curves. The blue curves indicate the closest wing position to the wall. These 
curve style and color code also apply for dimensionless normal and axial forces in Figs. 5-6, which were obtained using Kinematics I in Table 2. The onset of the timing is set to the first transition time-stop of ramp-type pitching maneuver for all experimental data presented.

$$
q_{p}=\rho U_{p}^{2} S / 2=\rho\left(c \alpha_{m}{ }^{\prime}\right)^{2} S / 2=\rho\left(2 K U_{\infty}\right)^{2} S / 2=4 K^{2} q_{U}
$$
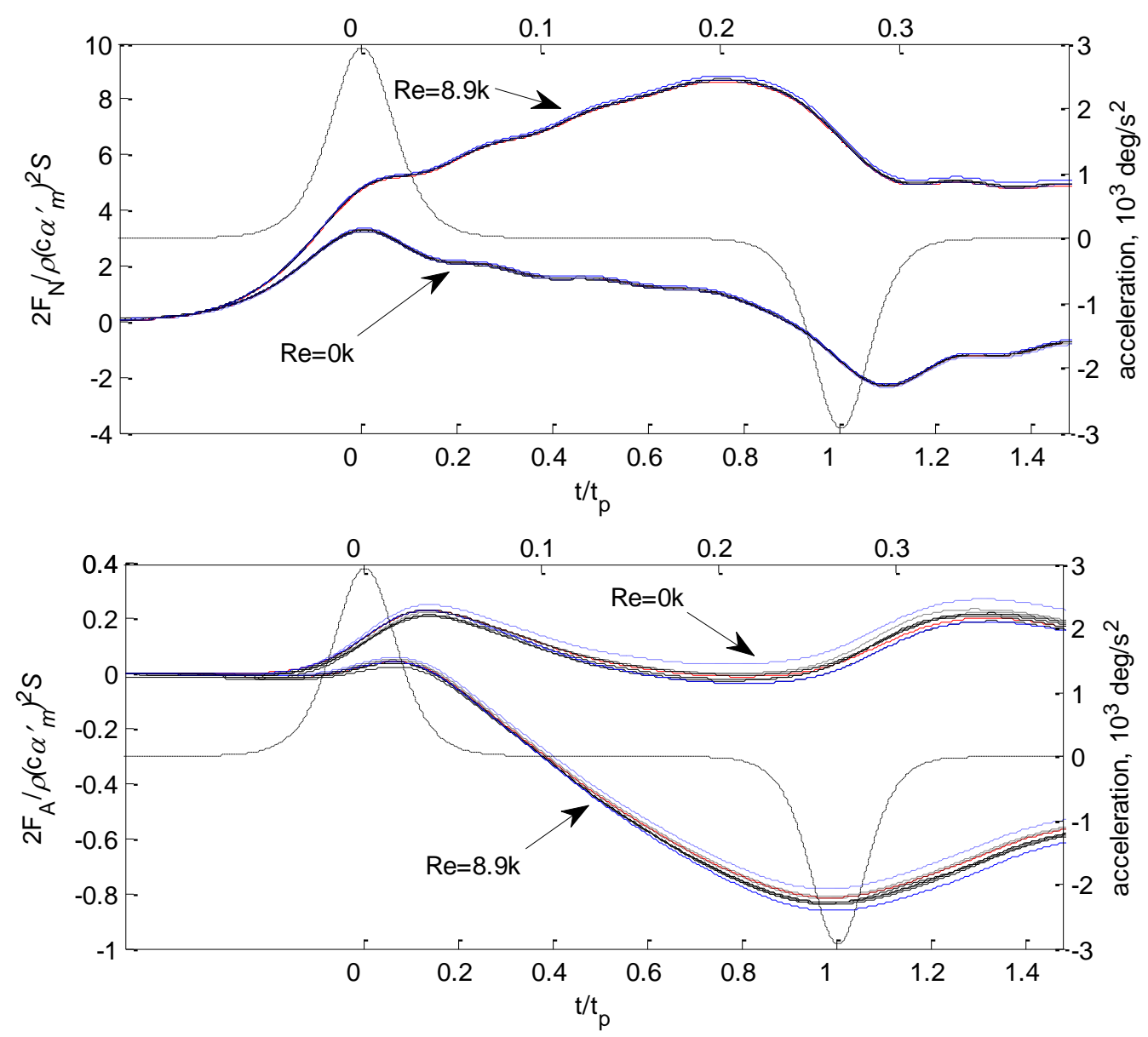

Fig. 5 (top) normal force and (bottom) axial force development as a function of pitch time of pitching wing at various crosswise positions in water channel.

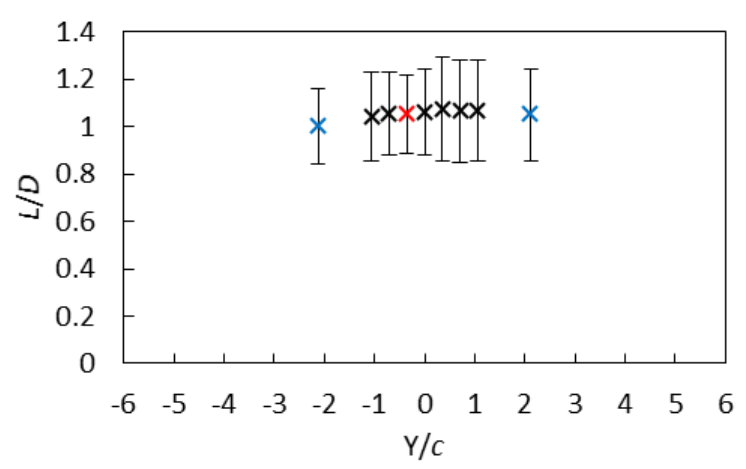

Fig. 6 Lift-to-drag ratio of stationary wing at various crosswise positions in water channel.

Figure 5 gives a comparison of force development as a function of pitching time among various wing initial positions in still water and the flow with $R e=8.9 \mathrm{k}$. The dynamic forces are scaled with a pressure force by maximum pitching speed $\left(U_{p}=c \alpha_{m}^{\prime}\right)$, instead of freestream velocity. This pitching-velocity pressure force $\left(q_{p}\right)$ is 
four times of reduced pitch rate squared of free-stream velocity pressure force $\left(q_{U}\right)$. As observed in Fig. 5, normal forces at different crosswise initial positions follow each other even flow conditions are different; similar tendency is also observed for axial force. The variations are within one standard deviation. Fig. 6 shows sidewall effect on stationary wing at 45-degree angle of attack; the discrepancy of forces at separate initial positions is negligible. Hereafter, we conclude that the effect of sidewall is insignificant, which is contrary to the computational result from two-dimensional pitching wing model at $K=0.2$ by Lian and $\mathrm{Ol}$ [28]. This observation suggests a wide range of applicable test area of the water channel using the wing with two-inch chord and two-chord span.

\section{B. Two-Dimensional PIV Image versus Stereo PIV Image}

Results presented in Figs. 7-8 are obtained using Kinematics II shown in Table 2 to disturb tranquil water, which maximum pitch rate $\alpha_{m}^{\prime}$ is 76.4 degree per second. There is a common feature among data, insignificant vorticity or out-of-plane velocity is in vicinity of the shadow except that the overlay regions of the wing. This common feature indicates the shadow introduces a level of noises within measurement uncertainty. Additionally, the vortical flow structure is similar between both cameras, as shown in Fig. 7, even though there are little variations on maximum and minimum values. The few variations are caused mainly by camera perspective at two separate locations and partly a result of the computational rounding.

Figures 7-8 show the flow development of the wing during pitching maneuver and early stage of holding at 45degree maximum angle of attack. During the pitching, two vortexes are present, a Starting vortex and a Pivot vortex. At early stage of the holding, there is another vortex present, which is a Stopping vortex. The maximum vorticity of these vortices and locations are presented in Fig. 9.

The Starting vortex contains positive vorticity and counterclockwise streamlines, forming at trailing edge. Considering the strength of the vortex is associated with its maximum vorticity detected in the flow field, we found that the Starting vortex obtains its strength at very early stage of the motion, reaches its full strength at 22.5 degrees on the way to maximum angle of attack, and then dissipates with pitch angle and time. The early process indicates the Starting vortex grows not just as a function of pitch acceleration, but pitch angle and time. Once the Starting vortex is fully developed and detaches the forming edge, it decades with time due to fluid viscosity. This later process is independent of pitch angle. According to Fig. 8, the Starting vortex initially has positive out-of-plane velocity around its center and then negative out-of-plane velocity is present instead. Both values of velocity are close to the maximum pitch speed. The positive out-of-plane velocity describes a flow moves spanwisely toward wingtip and out of the paper toward readers, the negative out-of-plane velocity indicates a spanwise flow moves toward wing root and into the paper from readers.

The Pivot vortex has negative vorticity surrounded by clockwise streamlines at pivot axis (i.e., leading edge in present study), which strength is weaker than the Starting vortex at same phase. As observed in Fig. 7 and 9, the Pivot vortex gains its strength at initial pitching motion, then gently grows in strength during constant pitch rate region, and then dissipates with time around pivot location until it is completely under the measurement uncertainty. The Pivot vortex is likely to stay at pivot location during the motion. Fig. 8 shows there is positive out-of-plane velocity nearby the center of Pivot vortex during the pitching motion. As the wing is pitched to maximum angle of attack, the out-of-plane velocity changes its direction to negative value whereas the Pivot vortex starts to lose its strength.

The Stopping vortex has negative vorticity and clockwise streamline just like Pivot vortex but present at trailing edge, as same as the forming edge of Starting vortex. As shown in Fig. 7, the Stopping vortex is unperceivable until the wing stops at maximum angle of attack and exhibit insignificant out-of-plan velocity. It looks likely that the Stopping vortex is formed by negative vorticities on upper surface, being generated during the pitching motion, sheds and swirls into a vortex downstream due to inertia force caused by wing deceleration. As observed in Fig. 8, these negative vorticities on upper surface are relative to the spanwise flow traveling toward wing tip. Once the Stopping vortex is formed, it drags the Starting vortex moving downward and pushes the Starting vortex downstream. The trajectories of the abovementioned vortexes are given in Fig. 9, which is obtained by detecting the maximum absolute values of the vorticity of the vortex and its location. 
Camera 1
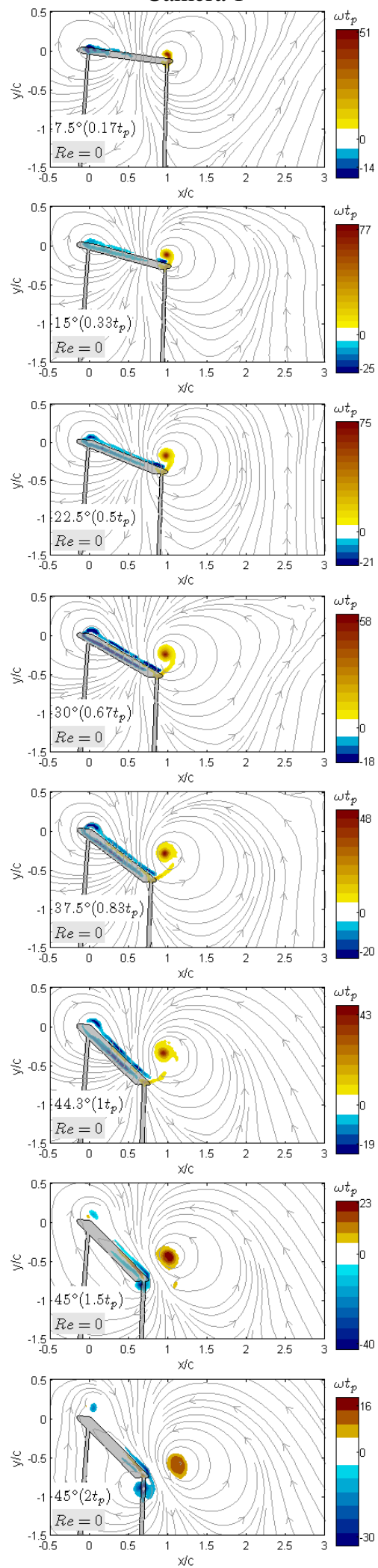

Camera 2
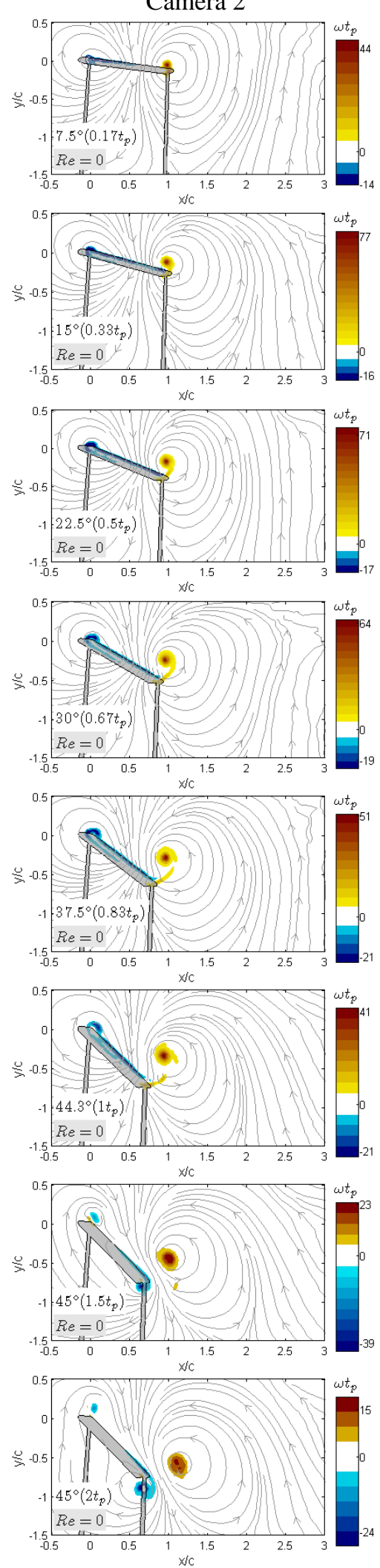
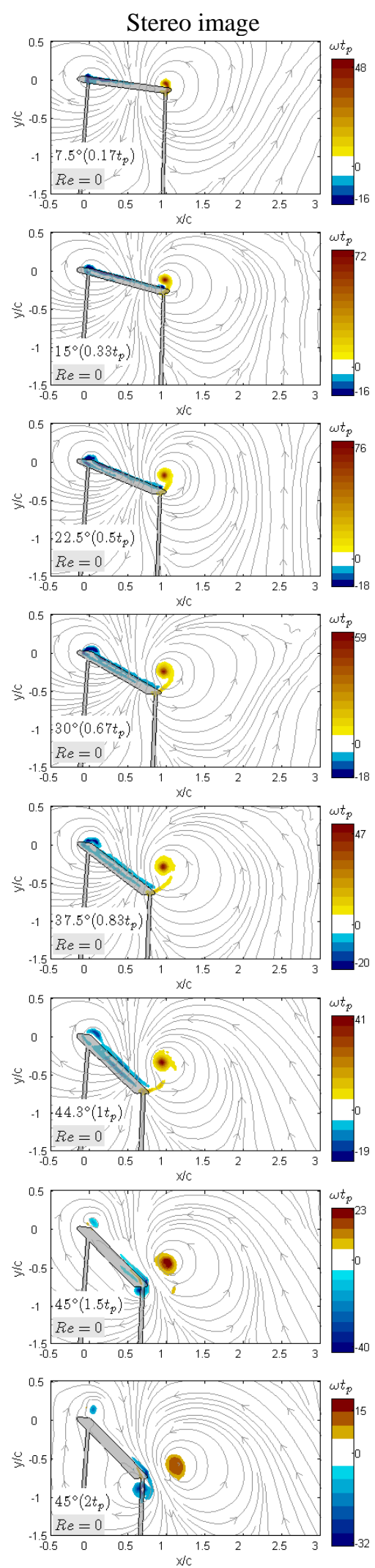

Fig. 7 Stereo images of vortical flow development by pitching wing in tranquil water. 

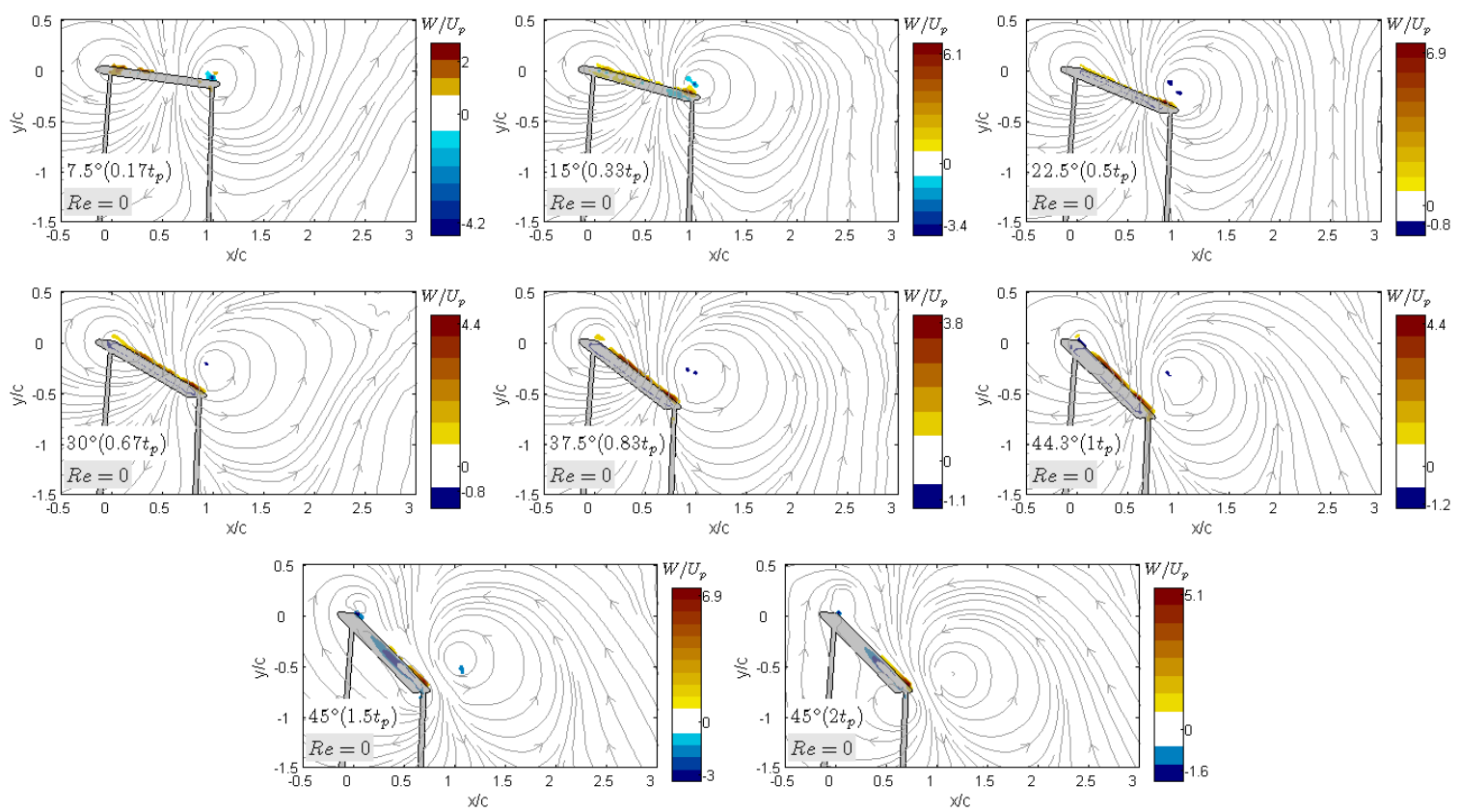

Fig. 8 Stereo images of out-of-plane velocity development by pitching wing in tranquil water.
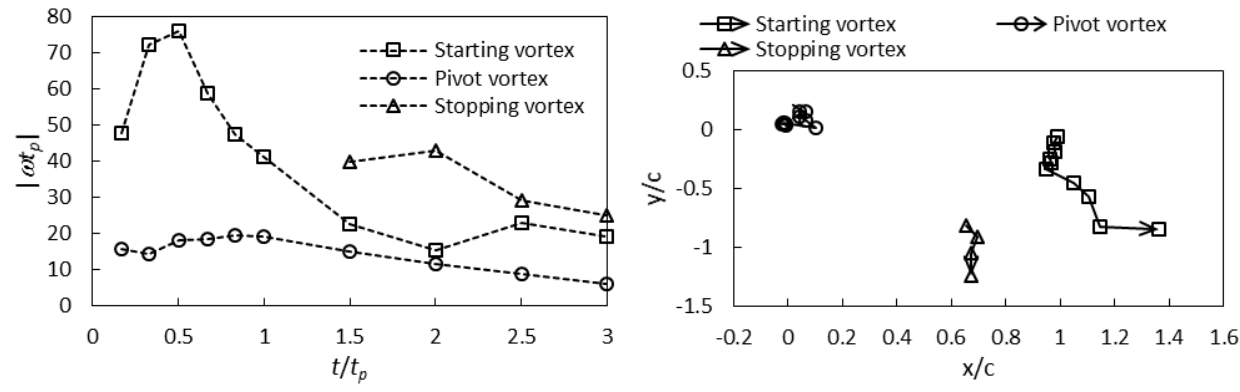

Fig. 9 Development of stereo vortexes on mid-span plane by pitching wing in tranquil water.

\section{Dynamic Flow of $K=0.39$}

In previous section, we examined data from two cameras and disclosed three vortex structures of pitching wing in tranquil water at mid-span, such as Starting vortex, Pivot vortex, and Stopping vortex. Now we are going to exam the dynamic flow for the case of $K=0.39$ at three span locations, mainly to discover the formation of tip vortex and its impact. Results documented here are based on Kinematics II in Table 2, same as that employed in previous section.

1) Pitching wing

Figure 10 shows similar flow patterns on the planes between both mid-span and three-quarter span, which involve the formations of a Starting vortex, a Leading-edge vortex, and a Secondary vortex, and the shedding of positive Trailing-edge vortex. Except the Secondary vortex, these vortexes promote the lift generation. As discovered by the case in still water, there is a Pivot vortex developing at leading edge during the motion; this Pivot vortex would superpose with typical Leading-edge vortex, contributing together to lift generation. The Secondary vortex is a vortex formed in the cavity underneath the neck of Leading-edge vortex, unperceivable for a pitching wing in still water. The presence of Secondary vortex likely slows down the progress of lift generation at angle of attack higher than 37.5 degree. At wing tip, only Starting vortex is present and lasts until 22.5-degree angle of attack. As pitch angle increases, the Starting vortex breaks down and mingles with negative vorticity layer on upper surface near trailing edge. The streamlines indicate the Starting vortex and the following Trailing-edge vortexes are stagnation points, where would satisfy Kutta condition. The negative vorticity layer on upper surface indicates a strong shear layer, which is thicker than the vorticity layer on lower surface; this phenomenon is also observed on 
the other two span locations. As revealed in Fig. 11, the negative vorticity layer is related to a strong spanwise flow adjacent to wing surface toward wingtip. On the plane of wingtip, there is another spanwise flow in vicinity of the lee of the wing.

Imaging there are particles being released from the freestream on the horizontal plane at wing tip, these particles take a detour around the edge of wingtip before hitting into it, then circulate about the tip edge toward leeward wing surface, and then moves along the wing surface toward the wingtip. The process encourages Tip vortex to be formed with pitch angle, especially during the motion. Fig. 11 shows that the formation of Tip vortex is constrained in the near wake before the place where Starting vortex is present, and under the plane of three-quarter span. The formation of Tip vortex contributes to the significant drag production.
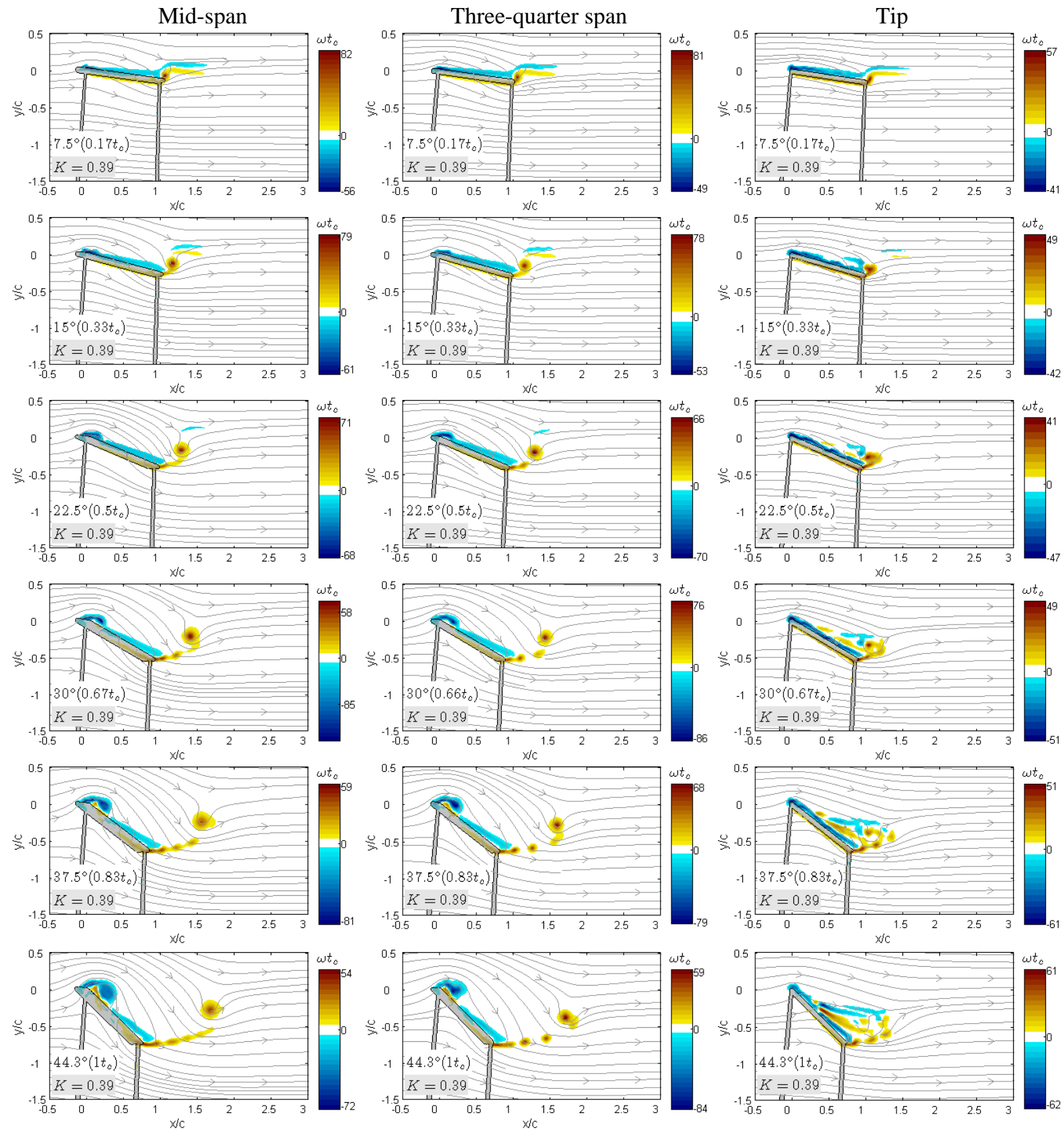

Fig. 10 Development of vorticity and aerodynamics by pitching wing at $K=0.39$. (a) Vorticity field. 

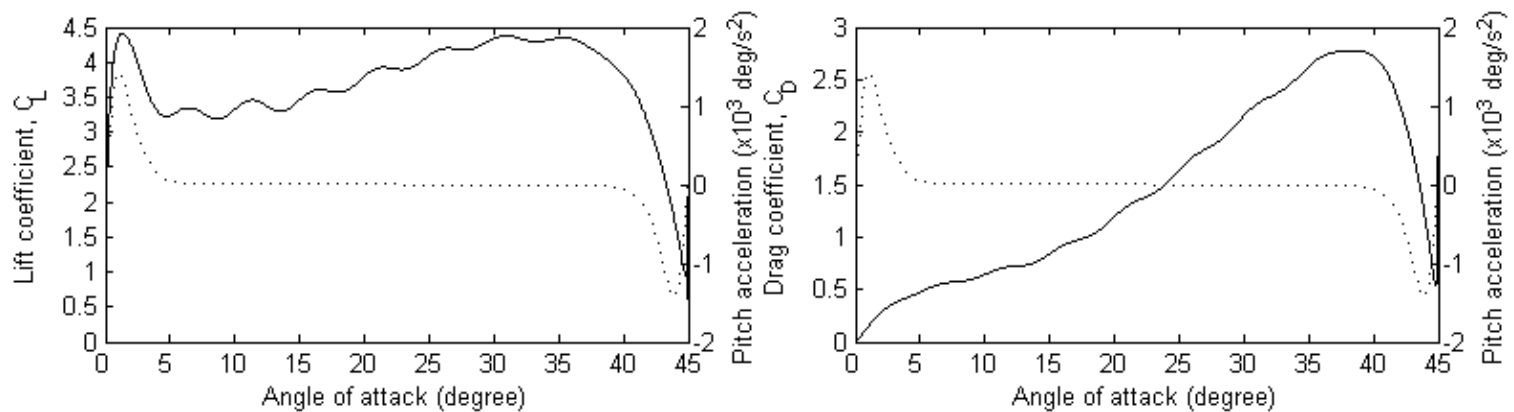

Fig. 10 (continued) Development of vorticity and aerodynamics by pitching wing at $K=0.39$. (b) Force coefficients as a function of angle of attack.

2) Stationary wing at 45 -degree angle of attack

As the wing is pitched to 45-degree angle of attack and held for a few convective times, as shown in Figs. 12-13, the transient flow behavior is expected before steady state condition is achieved. Unlike the flow structures shown in Figs. 10-11, the spanwise variations between the planes of mid-span and three-quarter span are significant. Taking the first glance on Fig. 12 without considering Starting vortex and Trailing-edge vortexes shed downstream, the size of the Leading-edge vortex on the plane at mid-span is greater than that at three-quarter span. Fig. 13 suggests the difference is caused by a spanwise flow induced by the Tip vortex formed during the motion.

The flow structures on the plane at mid-span remain intently two-dimensional, a large scale and round Leadingedge vortex is perceived during the early stage of the holding. After experiencing a process of elongation, the core of the Leading-edge vortex starts to break down at fourth convective times even though the neck of the Leading-edge vortex remains attached to the leading edge. As shown in Fig. 13, the breaking-down Leading-edge vortex must associate with the shedding of a positive out-of-plane velocity, which indicates a spanwise flow toward wing tip. This spanwise flow dies out within one chord after three convective times. Meanwhile, there exists another spanwise flow with negative sign present in the flow field. This spanwise flow initially goes with Leading-edge vortex, but then is trapped toward upper wing surface and then convected to the trailing edge. The strength of the negative spanwise flow at trailing edge corresponds to incoming formation of large-scale Trailing-edge vortex between phases of the fourth and seventh convective time. The weaker the negative spanwise flow, the stronger the largescale Trailing-edge vortex in formation. Considering the flow structure near the wake, the Stopping vortex is perceivable at a phase of the second convective time, and followed by the shedding of positive small-scale Trailingedge vortexes, and then the shedding of negative small-scale Trailing-edge vortexes. The former vortex shedding is relative to Secondary vortex but not to the growth of Leading-edge vortex. The out-of-plane velocities of these small scale vortexes are weak and under the measurement uncertainty. Moreover, as Starting vortex travels downstream with time follows the positive spanwise flow.

The flow structures on the plane at three-quarter span are significantly influenced by spanwise flows from wing tip. The Leading-edge vortex is elongated much earlier than that at mid-spane due to a more pronounced spanwise flow toward wing root, initiated at leading edge and convected on upper wing surface downstream with time. After the process of elongation, the core of Leading-edge vortex breaks down at third convective time; the breaking-down process is much earlier than that at mid-span. At same times, the positive spanwise flow presents, grows, and then fates at leading edge. Following the Stopping vortex in the near wake is a vortex street, and then comes a shedding of a positive Trailing-edge vortex originated from the wing tip at fourth convective time. Another formation of Leading-edge vortex is present in next following phases.

As the plane of wing tip is concerned, small-scale vortexes are scattered in the near wake at early phases of the holding. A significant positive Trailing-edge vortex is out of the sight until the third convective time; this positive Trailing-edge vortex sheds after the fifth convective time and dissipates quickly under measurement uncertainty in a half convective time. The formation of the positive Trailing-edge vortex is related to the negative spanwise flow adjacent, as show in Fig. 13. The swirl about wingtip, observed during the pitching motion, becomes weaker and disappears after the fourth convective time. 

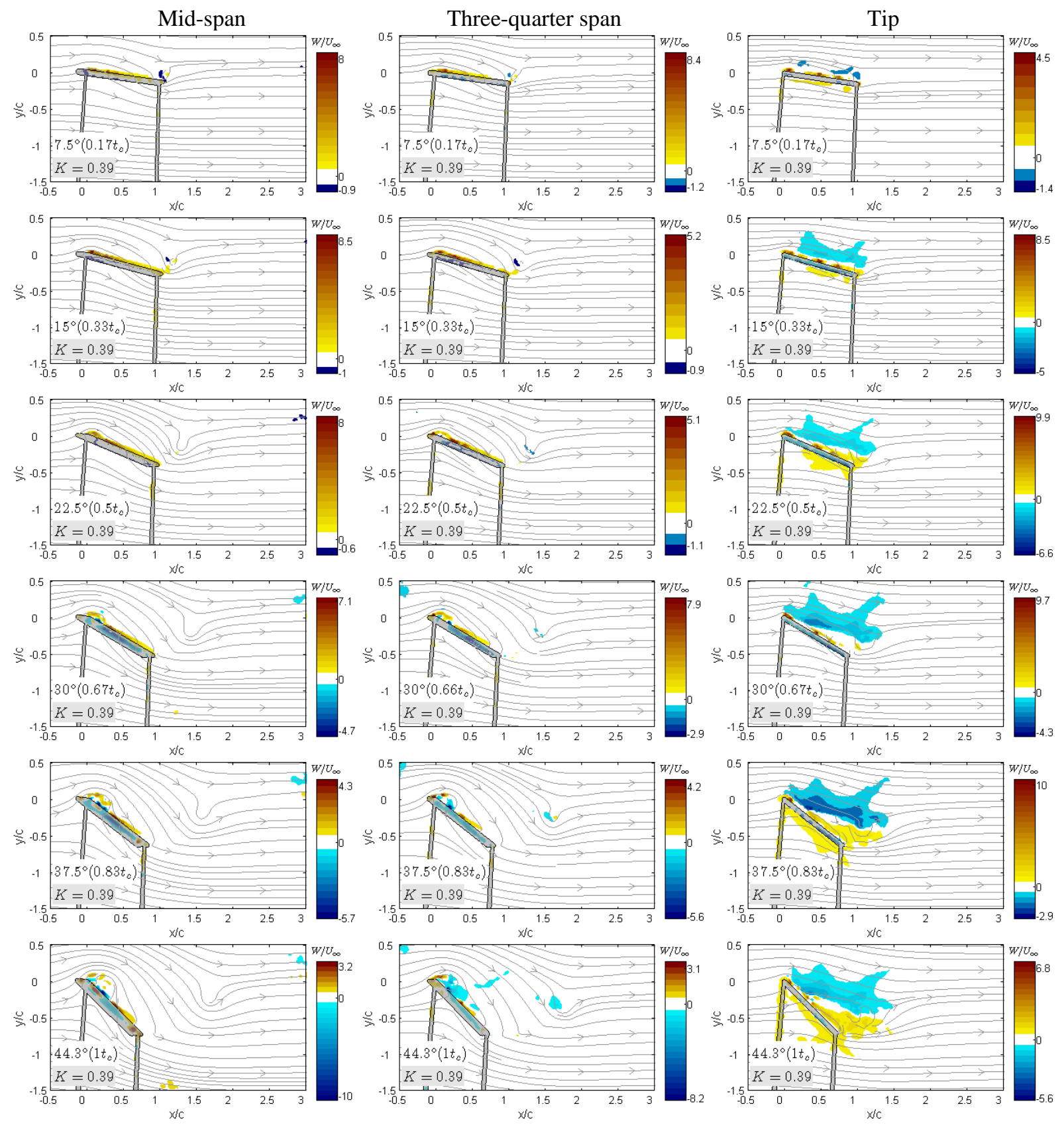

Fig. 11 Development of spanwise flow and aerodynamics by pitching wing at $K=0.39$. (a) Out-of-plane velocity field.

\section{Conclusion}

Reported in this study are dynamic flow fields by pitching a rectangular flat-plate wing in tranquil water and $K=$ 0.39 and early transient phases of stationary wing at 45-degree maximum angle of attack. Stereo images by a lensshift stereoscopic PIV system were constructed to capture particle velocity in three components at three span locations, along with data by direct force measurements to understand complex flow fields on unsteady force generation. The investigation on sidewall effect, by direct force measurement, ascertain its subtle influence on data by both wing and facility configuration presented in this paper. Conclusions are summarized below. 


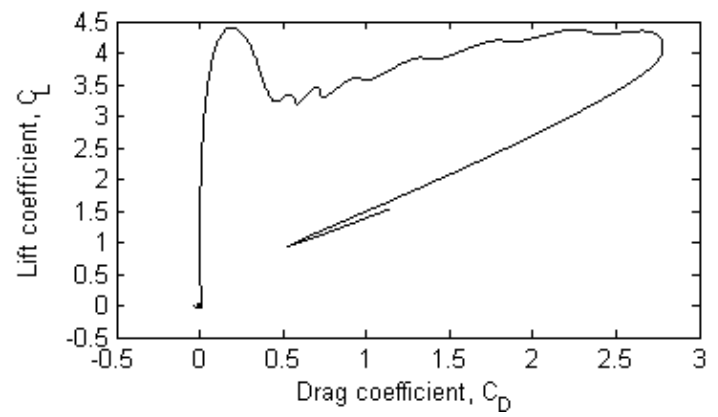

Fig. 11 (continued) Development of spanwise flow and aerodynamics by pitching wing at $K=0.39$. (b) Drag polar.

1. A Starting vortex is present at earlier phase of 7.5 degree in all span locations considered, which formation contributes a rapid force generation at onset of the motion regardless of freestream velocity. The Starting vortex at wing tip breaks down after 30-degree angle of attack in the free stream with $U_{\infty} \neq 0$.

2. The formation of a Tip vortex is present during the pitching motion in the free stream with $U_{\infty} \neq 0$, and is constrained between the wing and the Starting vortex, and below the three-quarter span. The Tip vortex introduces significant drag force to the wing.

3. A Pivot vortex together with typical Leading-edge vortex strengths the circulation over the wing and therefore enhance the force generation.

4. The transient flow by pitching wing held at 45 -degree angle of attack is driven by spanwise flow caused by Tip vortex, which decreases the aerodynamic forces. The flow features at mid-span is the shedding of positive Trailing-edge vortex and then negative Trailing-edge vortex. A vortex street is present at threequarter span. The shedding of a Stopping vortex and dissipation of Leading-edge vortex are common features between mid-span and three-quarter span.
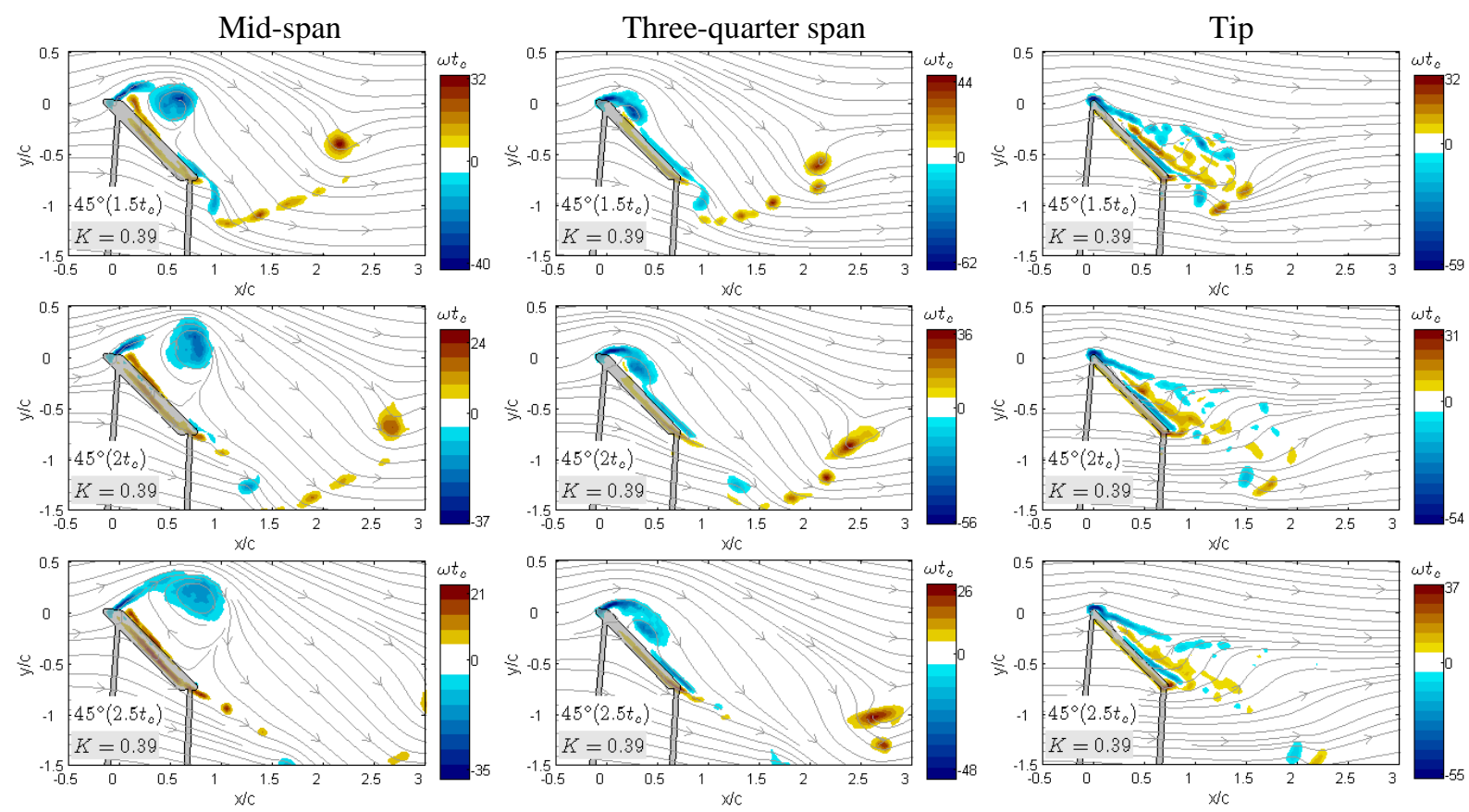

Fig. 12 Development of vorticity and aerodynamics by stationary wing at 45-degree angle of attack. (a) Vorticity field. 

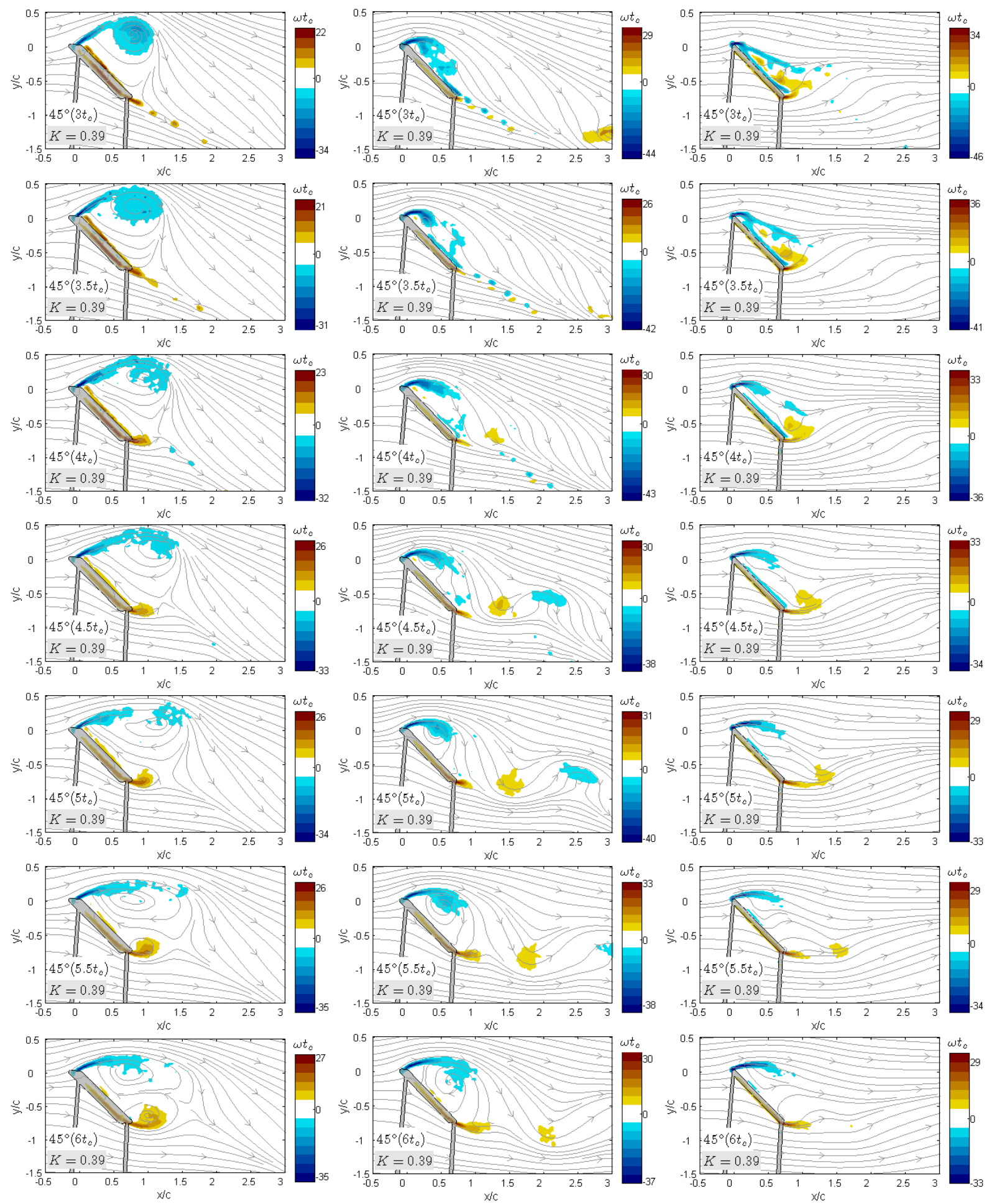

Fig. 12 (continued) Development of vorticity and aerodynamics by stationary wing at 45-degree angle of attack. (a) Vorticity field. 

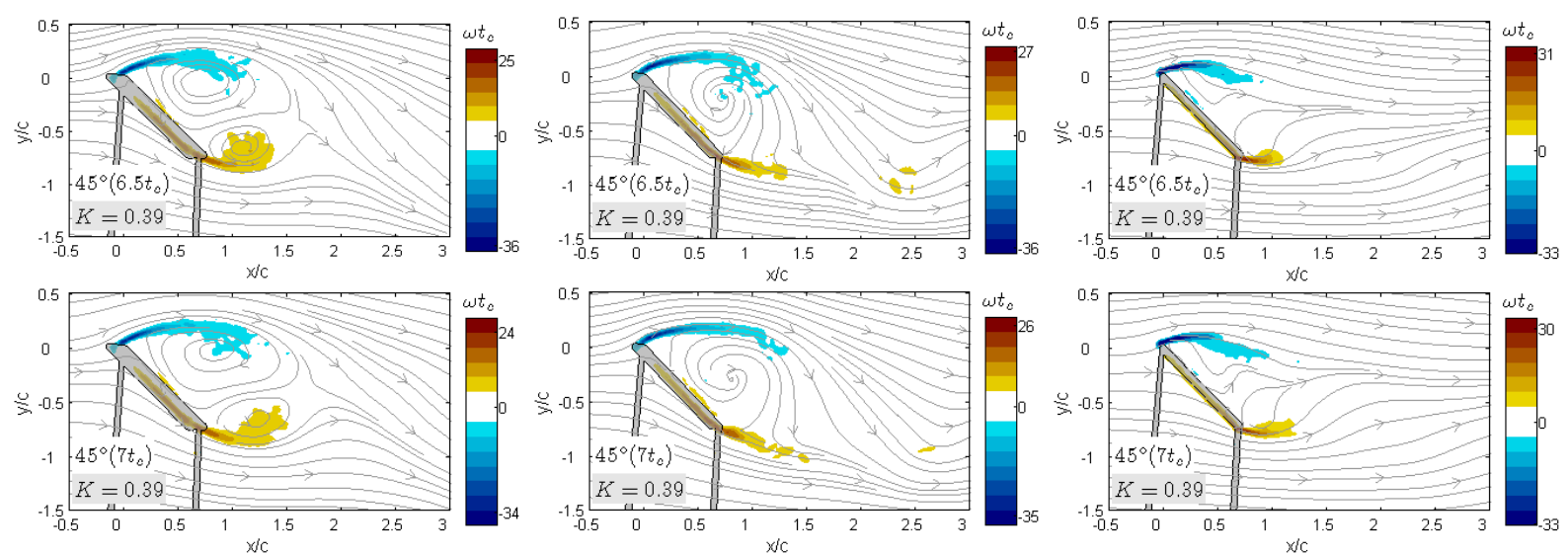

Fig. 12 (continued) Development of vorticity and aerodynamics by stationary wing at 45-degree angle of attack. (a) Vorticity field.

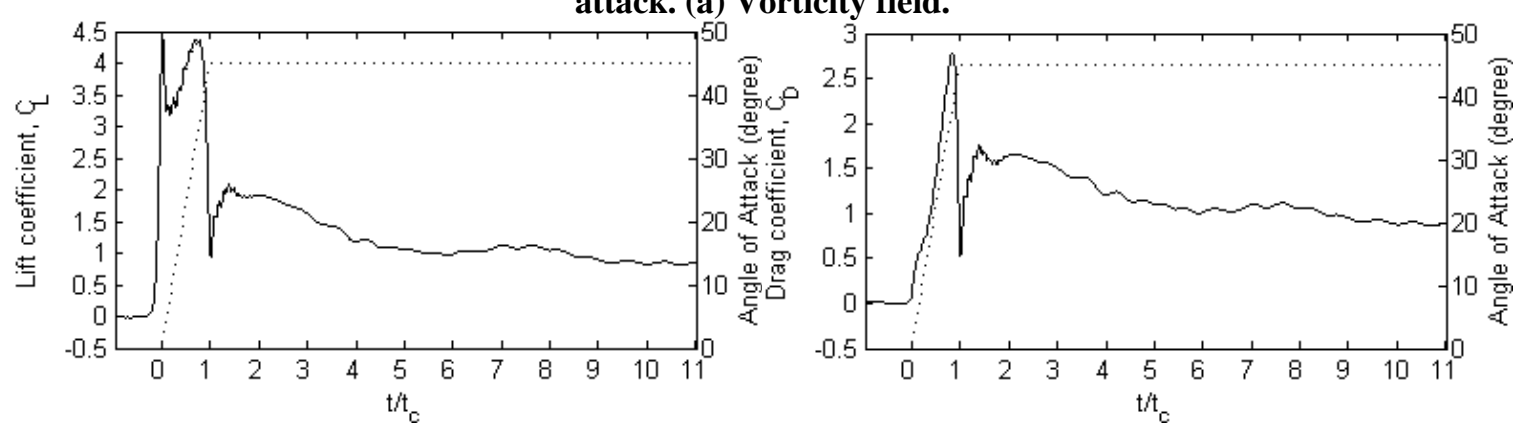

Fig. 12 (continued) Development of vorticity and aerodynamics by stationary wing at 45-degree angle of attack. (b) Force coefficients as a function of convective time.
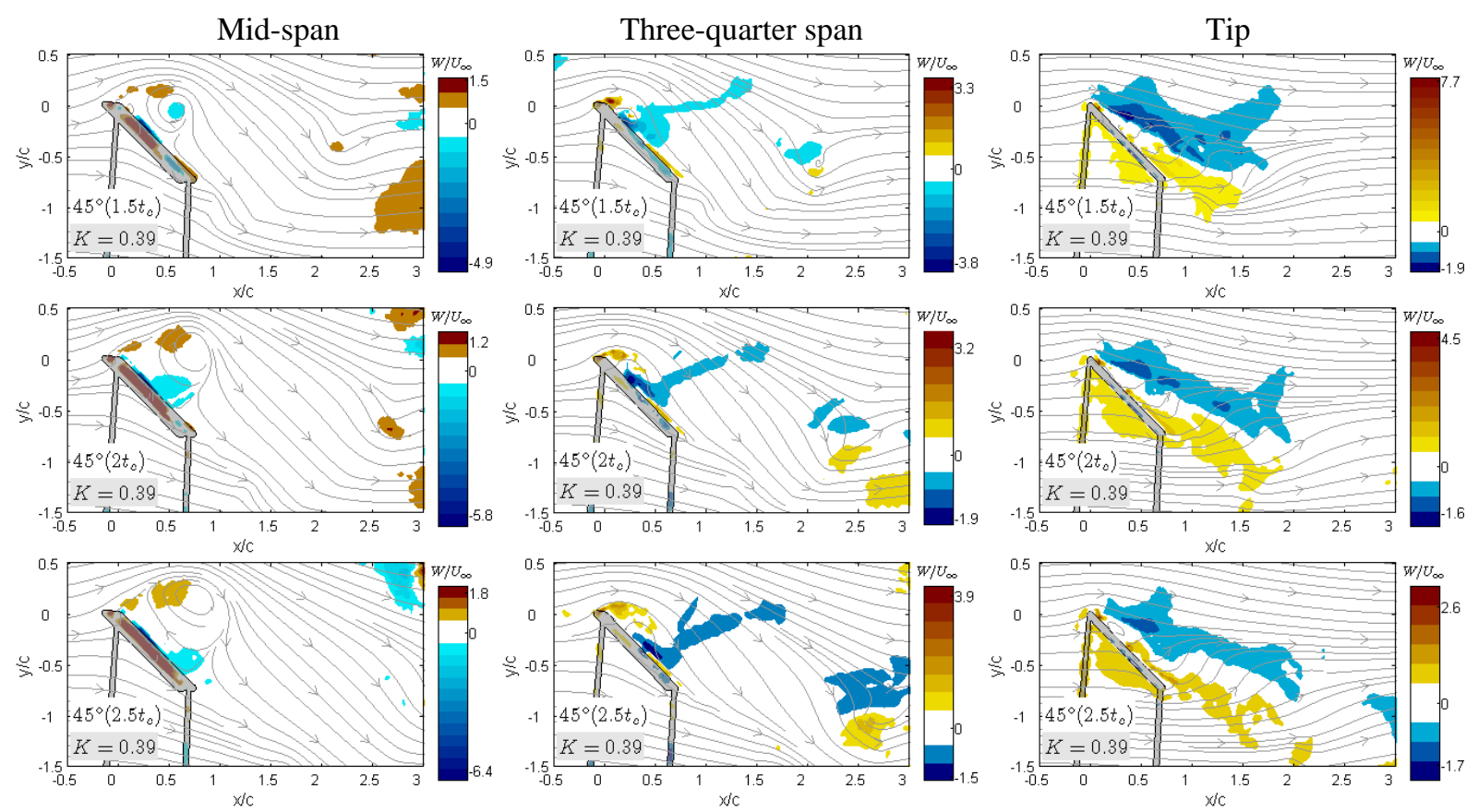

Fig. 13 Development of spanwise flow by stationary wing at 45-degree angle of attack. 

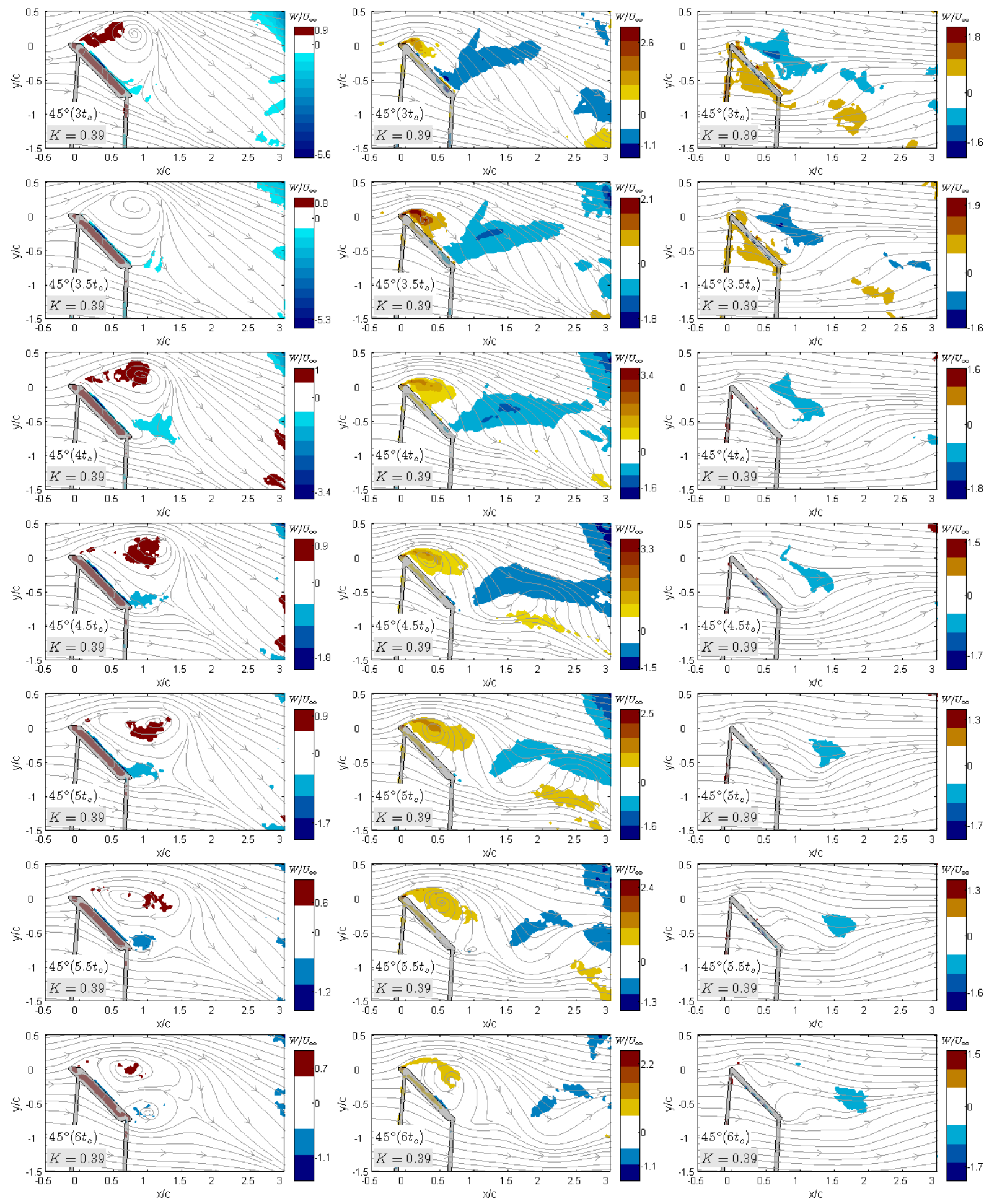

Fig. 13 (continued) Development of spanwise flow by stationary wing at 45-degree angle of attack. 

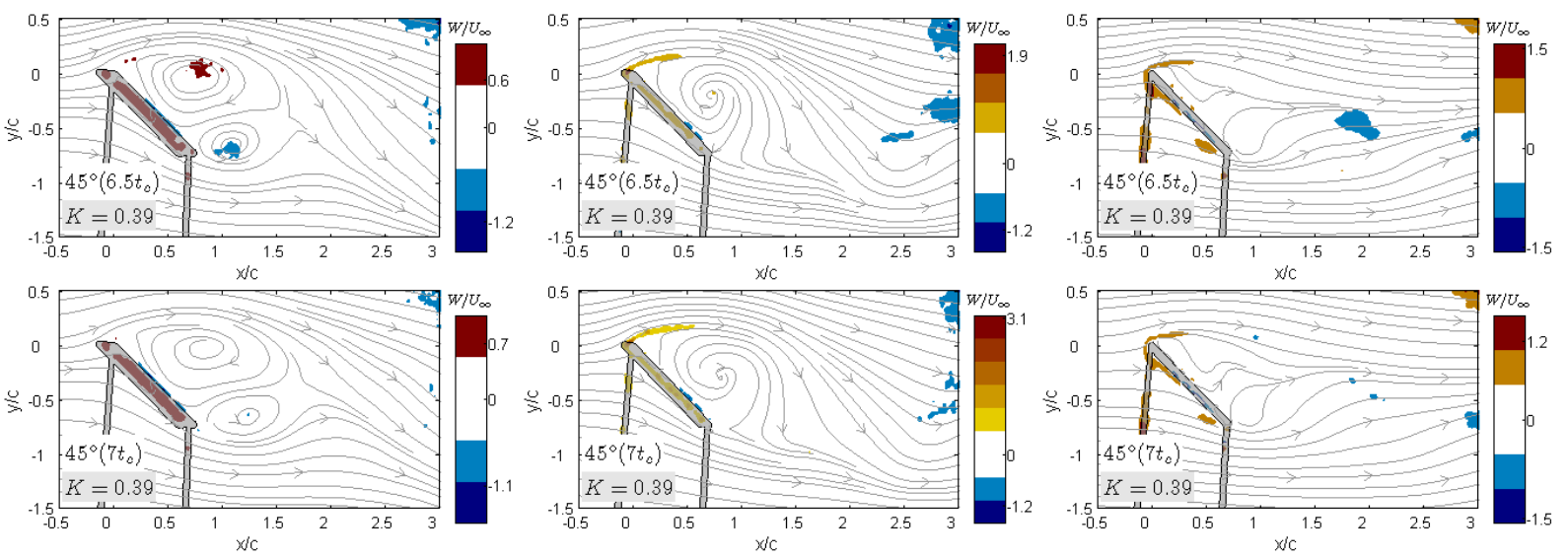

Fig. 13 (continued) Development of spanwise flow by stationary wing at 45-degree angle of attack.
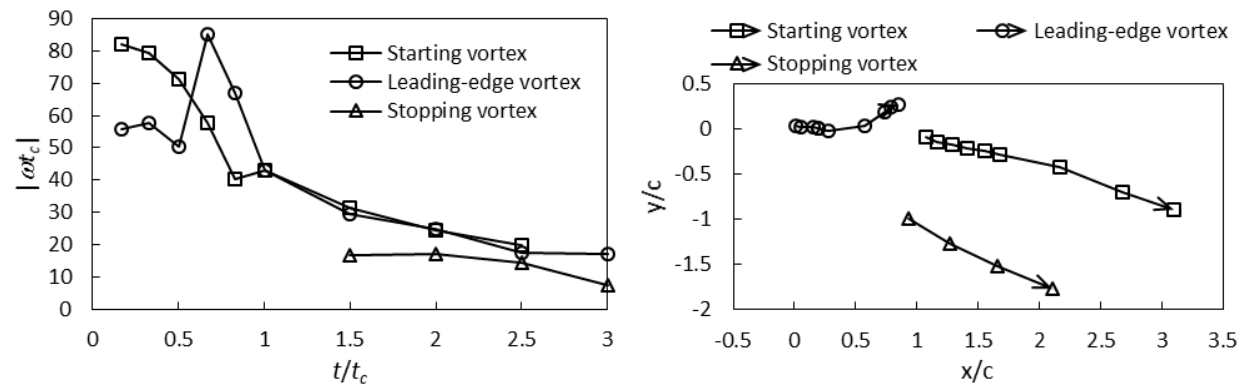

Fig. 14 Development of vortexes on mid-span plane by pitching wing at $K=0.39$.

\section{Acknowledgments}

The authors would like to thank Dr. Michael OL, Air Force Research Laboratory (AFRL) and Prof. Kenneth Granlund, North Carolina State University, for many fruitful discussions. The work was sponsored in part by the Air Force Office of Scientific, Multidisciplinary University Research Initiative (MURI), under Contract FA9550-07-10547, by the Michigan/AFRL Collaborative Center in Aeronautical Sciences, and by Ministry of Science and Technology, Taiwan under Contract MOST 104-2218-E-606-003.

\section{References}

${ }^{1 .}$ Strickland, J.H. and Graham, G.M., "Force Coefficients for a NACA-0015 Airfoil Undergoing Constant Pitch Rate Motions", AIAA Journal, Vol. 25, No. 4, 1987, pp. 662-624.

2. Graham, G.M. and Strickland, J.H., "An Experimental Investigation of an Airfoil Pitching at Moderate to High Rates to Large Angles of Attack", 24th AIAA Aerospace Science Meeting, AIAA-86-0008.

${ }^{3}$ Strickland, J.H. and Graham, G.M., "Dynamic Stall Inception Correlation for Airfoils Undergoing Constant Pitch Rate Motions", AIAA Journal, Vol.24, No.4, 1985, pp. 678.

4. Shih, C., Lourenco, L., Dommelen, L.V., Krothapalli, A., "Unsteady Flow Past an Airfoil Pitching at a Constant Rate", AIAA Journal, Vol. 30, No. 5, 1992, pp. 1153-1161.

${ }^{5}$ Walker, J.M. and Chou, D.C., "Forced Unsteady Vortex Flows Driven by Pitching Airfoils", 19th AIAA Fluid Dynamics, Plasma and Dynamics and Lasers Conference, AIAA-87-1331.

6. Walker, J.M., Helin, H.E., and Strickland, J.H., "An Experimental Investigation of an Airfoil Undergoing Large-Amplitude Pitching Motions", AIAA Journal, Vol.23, No.8, 1985, pp. 1141-1142.

7. Walker J.M. and Chou, D.C., "Unsteady Surface Pressure Measurements on a Pitching Airfoil", AIAA Shear Flow Control Conference, AIAA-85-0532.

8. Helin, H.E. and Walker, J.M., "Interrelated Effects of Pitch Rate and Pivot Point on Airfoil Dynamic Stall", 23rd AIAA Aerospace Sciences Meeting, AIAA-85-0130. 
9. Jumper, E.J., Dimmick, R.L., and Allaire, A.J.S., "The Effect of Pitch Location on Dynamic Stall", Transactions of the ASME, Vol. 111, 1989, pp. 256-262.

10. Jumper, E.J., Schreck, S.J., and Dimmick, R.L., "Lift-Curve Characteristics for an Airfoil Pitching at Constant Rate", Journal of Aircraft, Vol. 2, No. 10, 1987, pp. 680-687.

11. Daley, D.C. and Jumper, E.J., "Experimental Investigation of Dynamic Stall for a Pitching Airfoil", Journal of Aircraft, Vol. 21, No. 10, 1984, pp. 831-832.

12. Albertson, J.A., Troutt, T.R., and Kedzie, C.R., "Unsteady Aerodynamic Forces at Low Airfoil Pitching Rates", Archive Set 340, 1988, pp. 454-462.

13. Acharya, M. and Metwally, M.H., "Unsteady Pressure Field and Vorticity Production over a Pitching Airfoil", AIAA Journal, Vol. 30, No. 2, 1992, pp. 403-411.

14. Ol, M.O., "The High-Frequency, High-Amplitude Pitch Problem: Airfoils, Plates and Wings", 39th AIAA Fluid Dynamics Conference, AIAA 2009-3686.

15. Ol, M. V., Altman, A., Eldredge, J. D., Garmann, D. J., and Lian, Y., "Resume of the AIAA FDTC Low Reynolds Number Discussion Group's Canonical Cases,” AIAA Paper 2010-1085, Jan. 2010.

16. Eldredge, J. D., Wang, C., and Ol, M. V. "A Computational Study of a Canonical Pitch-up, Pitch-down Wing Maneuver," AIAA Paper 2009-3687, June 2009.

17. Granlund, K., Ol, M.V., Garmann, D., Visbal, M. and Bernal, L.P. "Experiments and computations on abstraction of perching", AIAA Paper 2010 - 4943, June 2010.

18. Granlund, K. O., Ol, M. V., and Bernal, L. P., "Experiments on Pitching Plates: Force and Flowfield Measurements at Low Reynolds Numbers," AIAA Paper 2011-872, Jan. 2011a.

19. Granlund, K. O., Ol, M. V., and Bernal, L. P., "Flowfield Evolution vs. Lift Coefficient History for RapidlyPitching Low Aspect Ratio Plates," AIAA Paper 2011-3118, June 2011 b.

20. Granlund, K. O., Ol, M. V., and Bernal, L. P., "Unsteady Pitching Flat Plates," Journal of Fluid Mechanics, Vol. 733, R5, 2013.

21. Baik, Y. S., Aono, H., Rausch, J. M., Bernal, L. P., Shyy, W., and Ol, M. L., "Experimental Study of a Rapidly Pitched Flat Plate at Low Reynolds Number,” AIAA Paper 2010-4462, June, 2010.

22. Yu, H.T., Bernal, L.P, and Morrison, C., "Experimental Investigation of Pitch Ramp-Hold-Return Motion of Flat Plates at Low Reynolds Number", AIAA Paper 2012 - 51, Jan. 2012.

23. Yu, H.T. and Bernal, L.P, "Effect of Pivot Point on Aerodynamic Force and Vortical Structure of Pitching Flat Plate Wings", AIAA Paper 2013 - 0792, Jan. 2013.

24. Visbal, M.R., "Three-Dimensional Flow Structure on a Heaving Low-Aspect-Ratio Wing", 49th AIAA Aerospace Sciences Meeting including the New Horizons Forum and Aerospace Exposition, AIAA 2011-219.

25. Yilmaz, T.O. and Rockwell, D., "Flow Structure on Finite-Span Wings due to Pitch-Up Motion", Journal of Fluid Mechanics, Vol. 691, 2012, pp518-545.

26. Anderson, J.D., Fundamentals of Aerodynamics, $5^{\text {th }}$ ed., McGraw-Hill, New York, 2011, Chaps. 4 - 5.

27. Leishman, J.G., Principle of Helicopter Aerodynamics, $2^{\text {nd }}$ ed., Cambridge University Press, New York, 2006, Chap. 8.

28. Lian, Y. and OL, M. V., "Computation and Experiments on a Low Aspect Ratio Pitching Flat Plate", 48th AIAA Aerospace Sciences Meeting Including the New Horizons Forum and Aerospace Exposition, AIAA paper 2010-0385.

29. Yu, H.-T., "Unsteady Aerodynamics of Pitching Flat Plate Wings." Ph.D. Dissertation, Aerospace Engineering Dept., Ann Arbor, University of Michigan, MI, 2014. 